\title{
Changements sémantiques sous l'influence de l'anglais: le cas de quatre « emprunts de sens » en français au Québec (1992-2012)1
}

\begin{abstract}
Résumé
Cette communication porte sur l'utilisation de quatre mots (alternative, opportunité, supporter, versatile) dont certaines acceptions sont considérées comme des emprunts de sens à l'anglais en français au Québec et qui ont pour caractéristique commune d'entrer en conflit, au plan axiologique, avec les acceptions françaises des mêmes mots. L’analyse comparative d'un corpus lexicographique (1880-2013) et de deux corpus québécois de français écrit (1992-2012) permet de montrer que les sens marqués comme anglais constituent les usages les plus fréquents de trois de ces mots. Les processus de changement sémantique qui permettent à ces sens de faire sens malgré l'ambiguïté introduite par l'opposition axiologique sont divers, mais signalent tous que les sens « anglais » ne sont pas si «étrangers» qu'ils le semblent de prime abord. L’adéquation des «équivalents » intralinguaux solution, occasion, appuyer et polyvalent est réévaluée au regard des résultats de l'analyse.
\end{abstract}

Mots-clés: emprunt sémantique, changement sémantique, français, anglais, Québec.

1 Cette contribution présente une partie des résultats obtenus dans le cadre de notre mémoire de maîtrise consacré à l'influence sémantique de l'anglais sur le français en usage au Québec (Paquet-Gauthier, 2015). Nous souhaitons remercier Bruno Courbon pour ses commentaires constructifs sur la version précédente de ce texte, ainsi que les éditeurs de ce volume pour leur travail hors pair. 


\section{Summary}

In this communication, we examine the use of four words (alternative, opportunité, supporter, versatile) considered to be English semantic loans in some of their uses in Québec French. These uses are caracterised by the fact that they conflict on an axiological level with some of the French meanings of the same words. Comparative analysis of a lexicographic corpus (1880-2013) and Québec written French corpora (1992-2012) shows that the meanings branded as "English" are the most commonly used. However, even though the axiological opposition they introduce results in semantic ambiguity, the various processes of semantic change that allow these meanings to make sense show that the "English" loans are not as foreign or borrowed as they seem. The appropriateness of intralingual equivalents such as solution, occasion, appuyer and polyvalent is re-evaluated on the basis of the results presented here.

Keywords: semantic loans, semantic change, French, English, Québec.

\section{Introduction}

Selon Valdman et al. (2005), les communautés qui vivent en situation de contact linguistique emploient davantage d'emprunts que les autres. En ce sens, la situation historico-politique du Québec fait de cette province canadienne un lieu privilégié d'observation du phénomène de l'emprunt en français, et en particulier de l'emprunt à l'anglais : la cohabitation ancienne et continue entre les communautés des deux langues officielles y favorise en effet l'utilisation de mots et d'expressions puisés dans la langue anglaise (Darbelnet, 1986 ; Poirier, 1994 ; Bouchard, 1998; De Villers, 2005).

Le contexte linguistique particulier du Québec se reflète dans des habitudes d'emprunts ${ }^{2}$ différentes de celles que l'on trouve dans d'autres régions de la francophonie : alors que les emprunts lexicaux sont prédominants en France, en Belgique et en Suisse, les emprunts de sens sont les plus usités au Québec (Mareschal, 1994 ; Klein et al., 1997). Précisons d'emblée que par emprunt de sens, nous entendons une acception d'un mot qui, dans le français en usage au Québec, semble avoir intégré des caractéristiques sémantiques propres à un mot de même étymon en langue anglaise. C'est le cas par exemple de alternative utilisé au sens de « solution de rechange». Comme ce type d'emprunt

2 Le terme emprunt sera toujours utilisé ici pour renvoyer à l'emprunt à l'anglais. 
n'affecte pas la forme du mot, il passe inaperçu pour la plupart des locuteurs, qui l'utilisent le plus souvent sans le savoir (Courbon \& Paquet-Gauthier 2014 : 149), même lorsque le sens ainsi emprunté prête à équivoque, voire entre en conflit avec les autres acceptions du mot auquel il se rattache. Il en est ainsi de versatile (« inconstant») qui prend, sous l'influence de l'anglais, le sens de «talentueux».

Les emprunts de sens sont historiquement les plus décriés dans la lexicographie corrective québécoise : comme le relèvent Lamontagne (1996) et Courbon \& Paquet-Gauthier (2014), ils sont entre autres accusés d'entraîner une « confusion ", une ambiguité sémantique qui « trompe » les locuteurs et menace la « clarté » de la langue. Malgré la présence importante de ce type d'emprunt tant dans les usages que dans les manuels correctifs, il demeure peu étudié - au Québec comme ailleurs - avec pour conséquence non seulement un certain flou terminologique en ce qui concerne les différentes modalités d'influence sémantique comme le calque, la traduction, les faux-amis, l'emprunt de sens, etc. ${ }^{3}$, mais également une méconnaissance des processus qui permettent à l'emprunt de sens de « faire sens » pour les locuteurs.

Les quelques études qui ont traité de l'emprunt de sens dans le langage courant (p. ex., Escaloya, 2000 ; Martel et al., 2001) ont recensé la proportion globale d'utilisation de sens « anglais » sur l'ensemble de l'utilisation d'un mot donné, sans distinction quant aux différentes acceptions ou à l'évolution de ces dernières dans le temps et dans les types de discours. L'emploi effectif des sens influencés de l'anglais dans l'usage ordinaire des locuteurs québécois demeure donc inconnu, et on ne sait rien des sens anglais réellement utilisés, les contextes dans lesquels ils le sont, ni comment ces nouveaux usages sémantiques (Courbon, 2007) se rattachent à la signification lexicale, en particulier lorsqu'ils semblent entrer en conflit avec elle. Ces questions sont pourtant essentielles dès lors que l'on cherche à connaître l'adéquation et la circulation des équivalents intralinguaux, comme c'est le cas dans les contextes d'aménagement linguistique.

Notre contribution a pour objectif d'apporter des éléments de réponse à ces questions grâce à l'examen, sur la période de 1992 à 2012, de l'utilisation de quatre mots traditionnellement identifiés dans la lexicographie comme des em-

3 Nous renvoyons les lecteurs aux textes de Colpron (1970), Humbley (1974), Darbelnet (1986), Chamizo-Dominguez \& Nerlich (2002), Loubier (2011) et Sablayrolles (2016) pour un tour d'horizon des différentes approches typologiques. Notons cependant qu'une véritable théorisation de l'influence sémantique interlingue reste à faire. 
prunts de sens (alternative, opportunité, supporter et versatile) et de celle de leur équivalent intralingual respectif (solution, occasion, appuyer et polyvalent) dans deux types de discours produits au Québec. Ces quatre mots ont en commun d'avoir acquis, sous l'influence de l'anglais, des caractéristiques sémantiques positives qui s'opposent à leur signification en français, plutôt négative. Les résultats de l'analyse permettent d'abord de rendre compte de l'utilisation réelle des emprunts de sens et des usages qui leur sont associés, puis de retracer quelques-unes des étapes des changements sémantiques « sous influence» qui semblent les différencier progressivement de leur équivalent. Nous proposons de mettre en lumière les différents processus de diversification sémantique des emprunts et les liens qui les rattachent aux sens «français » (ou considérés comme tels) des mêmes mots.

\section{Corpus et méthode d'analyse}

La méthode d'analyse s'appuie sur la comparaison entre la description lexicographique qui est faite des emprunts de sens et l'utilisation effective des mots auxquels ils se rattachent ${ }^{4}$. Dans ce qui suit, mot renvoie au signifiant, emprunt de sens (ES) à un sens marqué dans la lexicographie québécoise comme étant anglais, et équivalent à la traduction intralinguale proposée dans le corpus lexicographique pour remplacer le mot utilisé dans un emprunt de sens.

\subsection{Corpus lexicographique et sélection des mots}

La sélection des mots et des équivalents s'est faite en deux étapes, à partir du dépouillement d'un corpus lexicographique. Ce corpus est composé de 59 ouvrages en français publiés entre 1880 et $2013: 19$ dictionnaires normatifs ${ }^{5}$ qué-

4 Soulignons que le raccourci commode employé dans les lexicographies normative et proscriptive et qui consiste à présenter un mot comme étant un emprunt sémantique ("opportunité est un anglicisme sémantique »), conduit à des incohérences. Le problème est souligné par Walker (2013 : 155), qui a dû écarter de son étude d'emprunts à l'anglais le terme digital : ce mot, présenté sans contexte, a été jugé tout à fait français par les locuteurs, qui pensaient au sens « relatif aux doigts » et non à celui de "relatif au code numérique » comme le souhaitait l'auteur. De fait, pour identifier un sens " anglais ", il faut avoir accès au contexte - à l'environnement syntaxique et lexical, au thème, au type de discours, etc.

5 Normatif est utilisé au sens de Auroux (1994). 
bécois et français, et 38 dictionnaires ou recueils proscriptifs québécois, auxquels s'ajoutent deux dictionnaires en anglais utilisés pour vérifier que les sens « empruntés » existent bien dans cette langue 6 .

Les ES des quatre mots à l'étude appartiennent au vocabulaire courant, leur première attestation lexicographique est d'ancienneté variable, et ils sont décrits (et critiqués7) dans la plupart des ouvrages correctifs publiés au Québec faisant partie du corpus. Ils ont été sélectionnés parce qu'ils semblaient entrer en conflit sémantique avec d'autres sens des mots auxquels ils se rattachent. Le dépouillement du corpus lexicographique pour chacun d'eux (définitions, exemples, renvois, marques d'usage, reformulations «en français ", équivalents proposés, etc.) a permis de déterminer quels emplois sont considérés «français » ou « anglais " pour une période donnée. Les résultats de cet examen lexicographique diachronique sont présentés sous une double forme : une définition qui synthétise l'ensemble des définitions pour chaque $\mathrm{ES}^{8}$, et un mot ou un syntagme en petites majuscules comme forme courte.

L'inventaire des équivalents, des renvois et des reformulations a permis d'identifier l'équivalent monolexical le plus fréquemment proposé pour éviter l'usage des ES ou d'un ES en particulier. Une description générale servant de point de départ pour la comparaison avec les usages associés aux ES a été effectuée à partir des définitions de ces équivalents dans deux dictionnaires du corpus 9 .

Les résultats de cette première étape sont présentés dans le tableau 1. Exception faite de CIRCONSTANCE et de PERSPECTIVE pour alternative, et de POLYVALENT (en parlant d'un humain) pour versatile, tous les ES sont attestés à une date antérieure à la période couverte par les corpus de productions textuelles ${ }^{10}$.

6 La liste des ouvrages qui composent le corpus lexicographique est disponible en ligne au https://hal.archives-ouvertes.fr/hal-01494381/file/Bibliographie\%20 Lodz\%202016\%2C\%20Myriam\%20Paquet-Gauthier.pdf.

7 Notons au passage que les sens identifiés comme anglais ne sont jamais acceptés : même dans la lexicographie dite "descriptive ", les emprunts de sens sont toujours accompagnés d'une marque d'usage critiqué.

8 Faute d'espace, n'est présenté dans ce texte que la synthèse des sens « anglais »; des éléments des sens "français " sont présentés au besoin.

9 Le Nouveau Petit Robert (2012) et le Multidictionnaire de la langue française (2009), le premier publié en France et le deuxième au Québec.

10 Certains ES sont aussi décrits à des dates plus anciennes dans des publications ne faisant pas partie du corpus. C'est entre autres le cas pour opportunité, critiqué dès 1918 au sens de ocCAsion favorable (Étienne Blanchard, "Chronique du bon langage ", La Presse, 28 septembre 1918). 
Tableau 1. Emprunts sémantiques décrits dans le corpus lexicographique et équivalents monolexicaux les plus fréquents

\begin{tabular}{|c|c|c|c|}
\hline Mot & Emprunts sémantiques & 1ère att. & Équivalent \\
\hline \multirow{4}{*}{ alternative } & $\begin{array}{l}\text { TERME D'UN DILEMME « une des deux pos- } \\
\text { sibilités entre lesquelles on doit choisir » }\end{array}$ & 1913 & \multirow{4}{*}{ solution } \\
\hline & $\begin{array}{l}\text { SOLUTION UNIQUE « seule solution qui rend } \\
\text { possible la résolution d'une difficulté » }\end{array}$ & 1970 & \\
\hline & $\begin{array}{l}\text { SUBSTITUTION « choix de remplacement, de } \\
\text { rechange » }\end{array}$ & 1970 & \\
\hline & $\begin{array}{l}\text { POSSIBILITÉ « n'importe laquelle des pos- } \\
\text { sibilités offertes » }\end{array}$ & 1970 & \\
\hline \multirow{4}{*}{ opportunité } & $\begin{array}{l}\text { OCCASION FAVORABLE « situation qui } \\
\text { présente des avantages potentiels » }\end{array}$ & 1940 & \multirow{4}{*}{ occasion } \\
\hline & $\begin{array}{l}\text { CHANCE « situation favorable qui survient } \\
\text { fortuitement » }\end{array}$ & 1982 & \\
\hline & $\begin{array}{l}\text { CIRCONSTANCE « ce qui caractérise le } \\
\text { moment présent » }\end{array}$ & 1993 & \\
\hline & $\begin{array}{l}\text { PERSPECTIVE « projection positive impli- } \\
\text { quant un gain professionnel ou financier » }\end{array}$ & 1996 & \\
\hline \multirow{3}{*}{ supporter } & $\begin{array}{l}\text { ADHÉRER « adhérer aux prises de positions } \\
\text { d'un candidat/parti/groupe » }\end{array}$ & 1880 & \multirow{3}{*}{ appuyer } \\
\hline & $\begin{array}{l}\text { POURVOIR « mettre à la disposition de } \\
\text { quelqu'un ce qui est nécessaire à ses } \\
\text { besoins » }\end{array}$ & 1896 & \\
\hline & $\begin{array}{l}\text { ENCOURAGER «prendre le parti d'un athlète, } \\
\text { d'une équipe sportive » }\end{array}$ & 1978 & \\
\hline \multirow{6}{*}{ versatile } & $\begin{array}{l}\text { TALENTUEUX « qui démontre du talent dans } \\
\text { plusieurs domaines » }\end{array}$ & 1967 & \multirow{6}{*}{ polyvalent } \\
\hline & $\begin{array}{l}\text { OUVERT D'ESPRIT « qui fait montre } \\
\text { d'ouverture d'esprit » }\end{array}$ & 1967 & \\
\hline & $\begin{array}{l}\text { MULTIFONCTIONNEL « qui a plusieurs utilités " } \\
\text { (objet) }\end{array}$ & 1967 & \\
\hline & ÉRUDIT « qui s'intéresse à plusieurs sujets » & 1968 & \\
\hline & UNIVERSEL « qui s'adapte à tout » & 1982 & \\
\hline & $\begin{array}{l}\text { POLYVALENT « apte à accomplir plusieurs } \\
\text { tâches » (humain) }\end{array}$ & 2003 & \\
\hline
\end{tabular}




\subsection{Corpus de productions textuelles}

L'utilisation des quatre mots a été examinée systématiquement dans un ensemble de productions textuelles composé de deux corpus de genres différents. Le premier est un corpus de textes journalistiques québécois publiés en 1992, 1997, 2002, 2007 et 2012 ; il compte un total de 383 millions de mots répartis dans ces cinq sous-corpus. Le type de discours que l'on trouve dans le corpus journalistique ( $C$-Jr) est plutôt soigné. Le deuxième est un corpus de commentaires publiés sur le réseau Facebook entre 2009 et 2012, plus spécifiquement sur des pages publiques de programmes télévisés québécois ; il compte 38,5 millions de mots. Le type de discours que l'on trouve dans le corpus Facebook $(C-F B)$ est relativement spontané et se rapproche de la pratique orale. Ensemble, les deux corpus rassemblent 421,5 millions de mots ${ }^{11}$.

\subsection{Méthode d'analyse}

L'analyse des corpus de productions textuelles comprend deux étapes distinctes successives : la première dresse le portrait de l'utilisation des ES sur la période 1992-2012, et la deuxième permet d'évaluer l'adéquation sémantique des équivalents proposés pour éviter l'usage des ES.

Dans un premier temps, toutes les occurrences des quatre mots sont relevées à l'aide d'un programme d'extraction de données ${ }^{12}$ permettant de conserver le cotexte jusqu'à concurrence de 40 mots de chaque côté. Le nombre maximal d'occurrences analysées pour chaque mot est de 300 par sous-corpus, soit un total potentiel de 1800 occurrences chacun ${ }^{13}$. Dans l'ensemble, près de 5000 occurrences ont été analysées, sur les 19000 occurrences de ces

11 Les journaux et magazines qui composent le C-Jr sont les suivants : La Presse, Les Affaires, Le Soleil, Le Devoir, L’Actualité, Le Quotidien, Protégez-vous, Journal de Québec et Journal de Montréal. Le C-FB (Dion-Girardeau 2013) est constitué des pages publiques Facebook des émissions suivantes : TVA nouvelles, Salut Bonjour!, Occupation double, Star Académie, Un souper presque parfait, La galère, Qu'est-ce qui mijote?, Un gars le soir et Lance et compte.

12 Le programme informatique pour le C-Jr a été conçu par Hugo Mailhot et celui pour le C-FB par Samuel Dion-Girardeau ; nous les remercions vivement pour leur aide.

13 Ce nombre n'est pas toujours atteint, soit parce que le mot est peu fréquent, soit parce qu'un nombre élevé d'occurrences de «bruits » (mot employé comme nom propre, dans un lien URL, dans un syntagme ou une phrase en anglais, etc.) 
mots que comptent les corpus. Les résultats sont donc à interpréter en termes de proportion d'emploi ; le nombre d'occurrences brutes est donné à titre indicatif.

L'examen des occurrences permet d'effectuer un premier tri en trois catégories, soit d'abord toutes les occurrences que les éléments présents en contexte identifient comme un sens «français» (i.e., l'un de ceux décrits comme tels dans le corpus lexicographique), puis celles que le contexte permet de rattacher à l'un des ES décrits dans le tableau 1. La dernière catégorie sert à regrouper temporairement les occurrences de « sens ambigus » qui ne sont pas décrits dans le corpus lexicographique. Ces occurrences sont réanalysées pour mettre au jour d'éventuels éléments sémantiques communs à de nouvelles acceptions. Lorsque cette analyse ne permet pas de dégager le sens du mot en contexte, l'occurrence est dite de « sens indéterminé ». Les résultats de l'examen des occurrences dans les corpus de productions textuelles dressent le portrait de l'emploi des différents ES et permettent de mettre en lumière des changements sémantiques qui s'effectuent petit à petit.

Dans un deuxième temps, les définitions des équivalents recueillies dans le corpus lexicographique sont comparées aux différents emplois des ES relevés dans les productions textuelles. Des exemples tirés des C-Jr et C-FB servent à illustrer les éléments de comparaison (contextes d'utilisation, équivalence sémantique, etc.) et à évaluer l'adéquation des équivalents proposés, au regard des usages des ES observés dans les corpus de productions textuelles.

\section{Résultats et discussion}

Un fait étonnant ressort de l'examen global des mots alternative, opportunité, supporter et versatile dans les corpus de productions textuelles : mis à part supporter, les sens «français » comptent pour une minorité des occurrences de ces mots. La figure 1 permet de visualiser la proportion des occurrences d'emploi de sens «français », de sens temporairement « ambigus » et d'ES dans l'ensemble des corpus. Ces résultats globaux demandent cependant à être détaillés : nous nous intéressons dans ce qui suit aux occurrences d'ES et de sens «ambigus ».

a été éliminé, soit enfin, dans le cas de alternative, parce que les occurrences de l'adjectif féminin ont été écartées. 


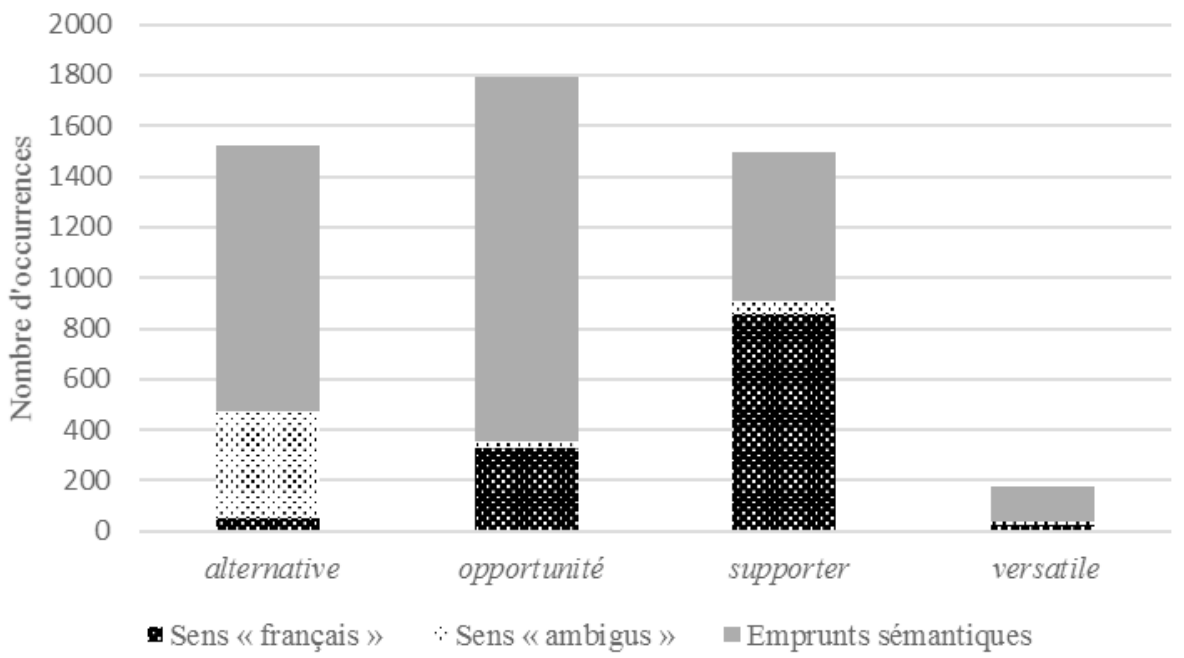

Figure 1. Proportion de sens « français » de sens « ambigus » et d'emprunts sémantiques dans les corpus de productions textuelles (C-Jr 1992-2012 + C-FB)

\subsection{Alternative}

Au total, 1521 occurrences du substantif alternative ont été analysées (tableau 2). De ce total, 27,7\% correspondent à des sens ambigus non décrits dans le corpus lexicographique, et seulement $3,4 \%$ à des sens français tel « situation où il faut choisir entre deux possibilités opposées », comme dans l'exemple (1) ${ }^{14}$ :

(1) Sous la houlette opportuniste de leur premier ministre, les Québécois s'en vont vers une alternative odieuse : ils auront à choisir entre une retraite humiliante (le report du référendum sine die) et un référendum tenu à contre-cœur [...]. (C-Jr 1992)

Les ES représentent donc la majorité des occurrences de alternative.

L'ES TERME D'UN DILEMME, dont l'attestation lexicographique est la plus ancienne pour alternative, ne représente que 3,2\% des occurrences et son emploi décroît entre 1992 et 2012, tout comme celui de SOLUTion UniQue (7,1 \%). Ces deux ES ont en commun avec le sens français en (1) une représentation de l'alternative comme un choix désagréable, une contrainte ou un dilemme. L'exemple (2) illustre le sens TERME D'UN DILEMME :

14 Exception faite de la mise en gras des caractères, les exemples ont été laissés tels quels. 
(2) [...] c'est pas nouveau le long terme pour les autos car les gens ont pas le choix s'ils veulent une auto neuve ou bien ils ont l'autre alternative, acheter usagé et toujours payer pour les bris et re et re et re. (C-FB)

L'ES substitution compte pour 14,2 \% des occurrences analysées, sans variation diachronique importante dans le C-Jr ; il est moins fréquent dans les usages spontanés du C-FB (8,9\%). Substitution sous-entend un deuxième choix, un «plan $B$ », un choix à défaut d'un autre qui lui serait préférable mais qui ne peut être sélectionné, comme dans l'exemple (3):

(3) [...] Mon premier choix est le hockey, mais je joue également à la crosse. Il s'agit d'une alternative au cas où je n'ai plus d'option. (C-Jr 1997)

L'ensemble des occurrences de ces trois ES à axiologie négative ne représente cependant qu'une minorité des emplois de alternative (total : 24,5\%). L'ES le plus fréquent dans les deux corpus est possibilité $(44,4 \%)$, avec peu de différence entre les discours soignés et spontanés. Dans son emploi le plus neutre, POssibILITÉ implique que l'alternative n'est qu'une des possibilités parmi un ensemble de choix plus ou moins acceptables. Toutefois, la proportion de ces emplois neutres diminue avec le temps tandis quaugmente celle des emplois « positifs » où alternative est accompagné d'un adjectif mélioratif comme bon, intéressant ou véritable, comme dans l'exemple (4):

(4) Il existe des produits logiciels libres, et de très bonnes alternatives à vos Windows et antivirus couteux, qui prennent leurs origines dans des produits open source... (C-FB)

L'augmentation de la fréquence de ce type d'emploi à partir de 2002 est notable à défaut d'être parfaitement linéaire, mais elle est surtout à mettre en parallèle avec le nombre important d'occurrences de sens ambigus correspondant aussi à un emploi où est absente la notion de dilemme constitutive de la signification de alternative en français (tableau 3 ).

Le réexamen des occurrences de sens ambigus permet en effet de dégager des contextes dans lesquels alternative est utilisé pour désigner un ou des choix implicitement considérés de valeur au moins égale à celle des autres choix proposés, sens que nous désignons par CHOIX ÉQUIVALENT, comme dans l'exemple (5) :

(5) Les actions à dividende élevé peuvent être une alternative aux titres à revenus fixes qui versent des intérêts. (C-Jr 2002) 

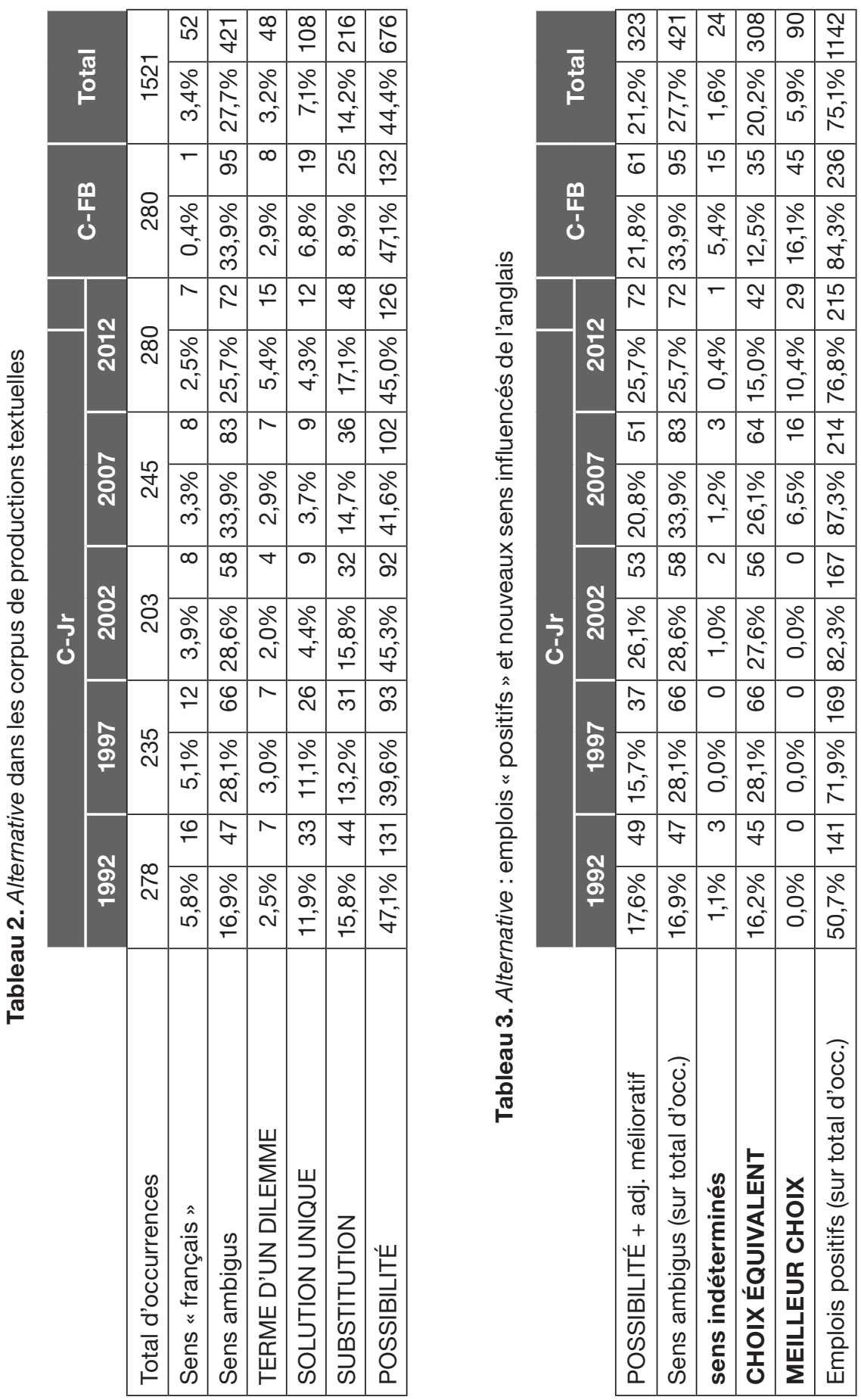
Mais on note surtout à partir du C-Jr 2007 l'apparition d'emplois dans lesquels alternative est un choix dont la valeur est intrinsèquement plus grande que celle des autres; ces emplois sont désignés par MEILLEUR CHOIX, comme dans l'exemple (6) :

(6) On va être le parti des intellos, une alternative au cow-boy Stephen Harper !

(C-Jr 2012)

Lauteur de ces lignes valorise manifestement davantage les « intellos » que les « cow boys ». MeIlleur choix représente 10,4\% des occurrences dans le discours soigné du C-Jr 2012 et atteint 16,1 \% dans le discours spontané (C-FB).

Globalement, les emplois " positifs » sont en croissance entre 1992 et 2012, et représentent $75,1 \%$ des occurrences ${ }^{15}$. Ni CHOIX ÉQUIVALENT ni MEILLEUR CHorx ne sont décrits dans les deux dictionnaires de langue anglaise consultés. Ces deux nouveaux sens s'inscrivent dans une suite de changements sémantiques ayant pour conséquence un renversement axiologique important, que l'on peut retracer au travers des différents ES décrits. De TERME D'Un DILEMME à SOLUTION UNIQUE, puis de SUBSTITUTION à POSSIBILITÉ, les usages sémantiques de alternative s'éloignent progressivement du dilemme et de la contrainte de choix pour prendre une valeur de plus en plus neutre, puis positive de liberté de choix - valeur fondamentale de la société occidentale actuelle. Même s'il est possible que CHOIX ÉQUIVALENT et MEILLEUR CHOIX soient également en usage en anglais malgré l'absence de description lexicographique et qu'on ne doit pas exclure l'hypothèse selon laquelle leur présence dans le français au Québec résulte d'un emprunt sémantique, il nous semble plus plausible qu'ils se soient développés en français, dans une succession de changements de sens imperceptibles, involontaires mais non pas arbitraires ${ }^{16}$ dont les différents ES décrits ci-dessus sont des témoins. Le terme emprunt, qui suppose un acte ponctuel et localisable, ne permet pas de rendre compte de ce processus diffus et continu d'influence sémantique.

15 La diminution de 10,5 \% observée entre le C-Jr 2007 et 2012 s'explique principalement par la reprise en 2012 d'un certain nombre d'articles où sont utilisés les ES TERME D'UN DILEMME et SUBSTITUTION par trois quotidiens différents appartenant au même groupe de presse, ce qui fait augmenter la proportion de ces ES dans le corpus.

16 Nous suivons en cela la théorie continuiste de l'évolution sémantique de Nyckees (2006). 
Le changement de polarité observé rend difficile de remplacer alternative par solution, l'équivalent monolexical le plus fréquemment proposé pour éviter l'emploi d'un ES : comme le suggère la description lexicographique qui en est faite, solution s'emploie dans des situations où un problème exige une résolution. Problème est d'ailleurs un cooccurrent très fréquent, comme dans l'exemple (7):

(7) "Filtrer" les gens qui ont des maladies psychiatriques n'est pas une solution à ce problème. (C-FB)

Lalternative n'est pas nécessairement liée à un problème : alternative et problème sont d'ailleurs rarement cooccurrents et lorsqu'ils le sont, l'alternative ne constitue pas une solution, comme le montre l'exemple (8):

(8) Le problème c'est qu'il y a pas d'alternative! (C-FB)

Ainsi, l'équivalent solution est-il de moins en moins adéquat pour remplacer alternative utilisé dans des sens considérés comme " anglais », puisqu'il sous-entend l'existence d'un problème à résoudre, tandis que la majorité des emplois de alternative renvoie à un ensemble d'options permettant une certaine liberté de choix.

\subsection{Opportunité}

Ont été analysées 1793 occurrences de opportunité. Globalement, 18,4 \% de ces occurrences correspondent au sens français « caractère de ce qui est à propos », comme dans l'exemple (9) :

(9) Interrogé sur l'opportunité de redémarrer le service intermodal (remorques sur rail), M. Fillion n'est pas très chaud à cette idée. (C-Jr 1997)

Toutefois, la fréquence de ce sens diminue radicalement dans le discours soigné du C-Jr, passant de 39,0 \% en 1992 à 7,8 \% en 2012, et il est totalement absent du discours spontané du C-FB, ce qui fait que même dans les usages soignés, ce mot est utilisé dans un sens « anglais » plus de neuf fois sur dix en 2012.

L'ES OCCASION FAVORABLE est l'acception la plus fréquente avec $64,8 \%$ du total et une croissance de plus de $30 \%$ dans le C-Jr. Au plan sémantique, cet ES représente l'opportunité comme devant être saisie afin d'en retirer les bénéfices, comme dans l'exemple (10): 
(10) Écho similaire de l'Union européenne pour laquelle l'accord de La Mecque est clairement une opportunité : «l'unité des Palestiniens ne pouvant qu'aider à la stabilisation de la situation et après, au processus de paix » [...]. (C-Jr 2007)

Cet ES se trouve souvent en cooccurrence avec des verbes comme saisir, offrir, donner ou manquer, et, de façon de plus en plus marquée, avec des adjectifs qui en soulignent le caractère positif ${ }^{17}$ tels que grande, belle, unique ou meilleure, comme en (11):

(11) Nous avons cette incroyable opportunité et nous ne l'utilisons pas. (C-Jr 1992)

Il est d'ailleurs remarquable que, peu importe le sens dans lequel il est utilisé, opportunité n'est jamais accompagné d'un adjectif négatif (?mauvaise opportunité, ?pire opportunité, etc.) dans les corpus. Le tableau 5 montre la croissance de la cooccurrence d'adjectifs positifs avec OCCASION FAVORABLE, qui a triplé entre 1992 et 2012.

Bien que l'ES CiRCONSTANCE ait été décrit récemment dans le corpus lexicographique (1993), aucune occurrence de ce sens n’a été relevée dans les corpus.

L'ES CHANCE ne compte que pour 2,6\% des emplois de opportunité. Au plan sémantique, cet ES se caractérise par le fait que les bénéfices surviennent fortuitement, sont « donnés » sans qu'il soit nécessaire d'engager une action en ce sens. On le relève le plus souvent dans des expressions telles avoir l'opportunité / une opportunité comme dans l'exemple (12):

(12) Donner des photos de la reine et de son mari, c'est comme si on avait l'opportunité d'avoir des photos de Jean Charest... Gratuitement ou pas, avec moi, elles iraient toutes à la poubelle, déchirées en milles $<$ sic $>$ morceaux! (C-FB)

L'ES PERSPECTIVE représente 12,9\% del'ensemble des occurrences analysées et sa fréquence a plus que doublé entre les C-Jr 1992 et 2012. Cet ES concerne explicitement une projection positive ou un avantage professionnel ou financier ; il est donc fréquent dans des contextes liés au domaine spécialisé de la finance, ce qui explique sans doute qu'il apparaisse peu dans le C-FB (7,0\%), où les sujets relèvent plutôt des variétés. Le tableau 6 présente les principaux syntagmes dans lesquels se réalise cet ES.

17 Notons cependant que le latin opportunitas « chance de faire quelque chose, condition favorable » dont est issu opportunité présentait déjà cette valeur positive. Le sens OCCASION FAVORABLE était d'ailleurs décrit sans aucune marque d'usage dans les éditions 1 à 8 du Dictionnaire de l'Académie; ce n'est qu'à partir de la $5^{e}$ édition que l'acception "qualité de ce qui est opportun » fait son apparition, et qu'avec la $9^{e}$ édition que le sens « occasion favorable » devient critiqué. 

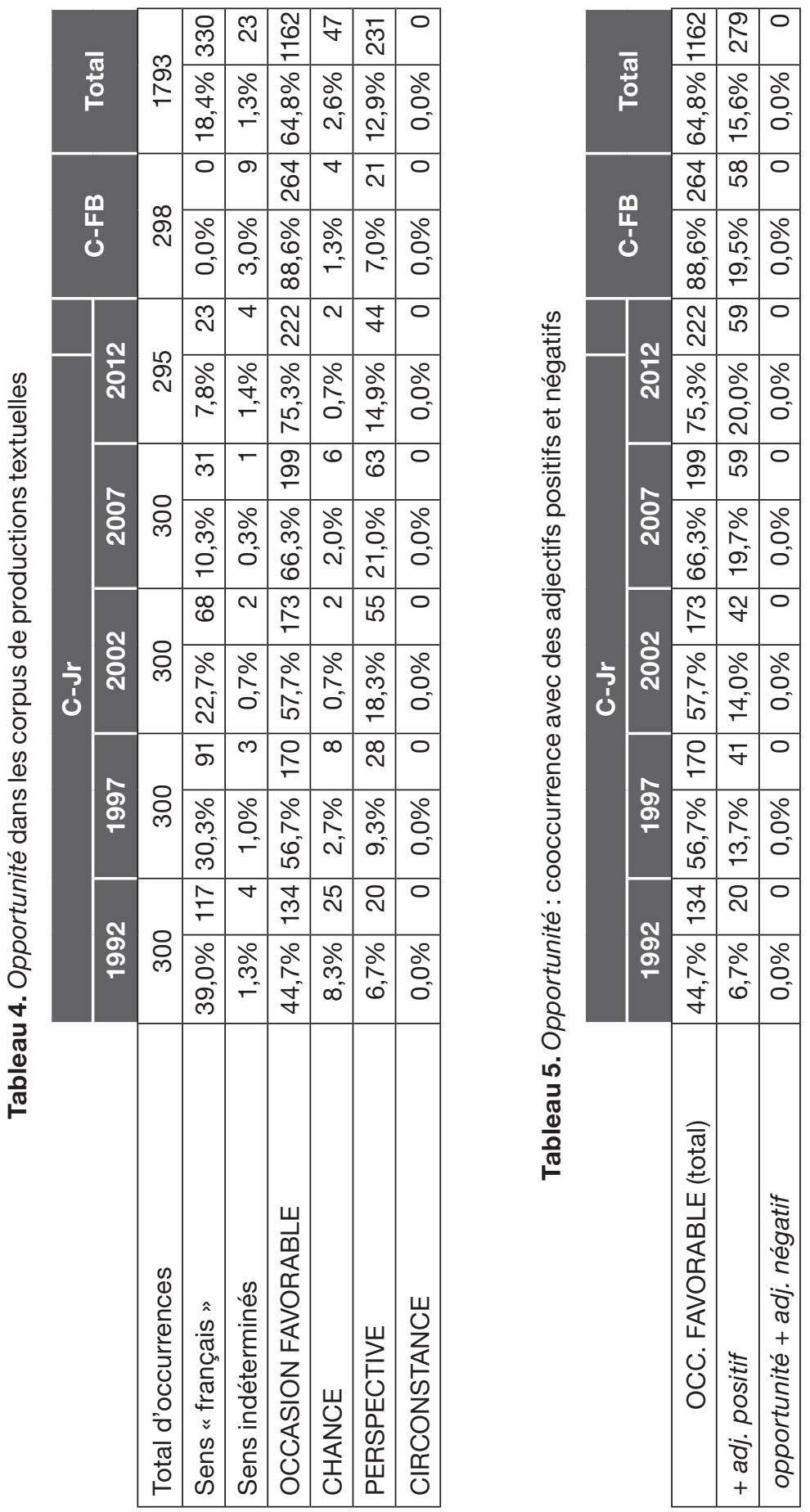
Tableau 6. Opportunité : principaux syntagmes pour l'ES PERSPECTIVE

\begin{tabular}{|c|c|c|c|c|c|c|c|}
\hline & \multicolumn{5}{|c|}{ C-Jr } & \multirow{2}{*}{ C-FB } & \multirow{2}{*}{ Total } \\
\hline & 1992 & 1997 & 2002 & 2007 & 2012 & & \\
\hline $\begin{array}{r}\text { PERSPECTIVE } \\
\text { (en nombre d'occurrences) }\end{array}$ & 18 & 31 & 52 & 58 & 44 & 21 & 218 \\
\hline coût d'opportunité & 1 & 2 & 3 & 3 & 2 & 1 & 12 \\
\hline étude d'opportunité & 4 & 2 & 1 & 4 & 2 & 0 & 13 \\
\hline fenêtre d'opportunité & 1 & 1 & 3 & 5 & 9 & 1 & 20 \\
\hline opportunité d'affaire & 9 & 4 & 10 & 13 & 13 & 3 & 52 \\
\hline $\begin{array}{r}\text { opportunité d'emploi / } \\
\text { carrière / travail }\end{array}$ & 3 & 6 & 10 & 1 & 3 & 8 & 31 \\
\hline $\begin{array}{r}\text { opportunité économique / } \\
\text { d'investissement }\end{array}$ & 0 & 3 & 8 & 6 & 0 & 0 & 17 \\
\hline autre & 0 & 13 & 17 & 26 & 15 & 8 & 79 \\
\hline
\end{tabular}

La diminution de la fréquence de ces syntagmes entre les C-Jr 2007 et 2012 (de 58 à 44 occurrences) s'accompagne de l'apparition de contextes où opportunité est utilisé sans complément et dans le sens de « emploi, avancement professionnel », comme en (13) :

(13) Boies a été laissé sur la touche à deux occasions lorsque le Rouge et Or de l'Université Laval se cherchait un coordonnateur offensif en 2010 et 2011. «J'aurais aimé obtenir une opportunité à Laval, mais je ne garde pas d'amertume ", a mentionné l'ancien maraudeur du Rouge et Or. (CJr 2012)

Bien que ce sens soit attesté dans les deux dictionnaires de langue anglaise consultés ${ }^{18}$, tous les exemples qui en sont donnés montrent opportunity accompagné d'un complément (job / employment / career opportunity); il semble donc plausible que l'emploi sans complément de opportunité PERSPECTIVE résulte d'une évolution sémantique indépendante en français.

Si l'on tient compte du fait que la fréquence du sens français « caractère de ce qui est à propos » et de l'ES CHANCE est en décroissance marquée entre 1992 et 2012, que les occurrences de OCCASION FAVORABLE et la cooccurrence de opportunité avec des adjectifs mélioratifs sont en augmentation, et que l'emploi de PERSPECTIVE est aussi en croissance, on peut conclure à un changement sé-

18 Merriam-Webster, sous opportunity: " promotion or elevation to a higher rank or position ». Oxford English Dictionary, sous opportunity: " a chance for employment or promotion; a job vacancy ». 
mantique dans lequel l'opportunité semble être de moins en moins associée à la chance et de plus en plus à un événement qui nécessite une action pour en tirer des avantages. Même s'il paraît probable que ce changement vers une signification axiologiquement plus positive ait été impulsé par l'anglais, il ne faut pas écarter la possibilité que l'influence de cette langue soit moindre que celle de la société occidentale moderne qui, en valorisant l'entrepreneuriat et l'action individuelle ( $c f$. Lahire 2004), fournit un climat propice ${ }^{19}$ à la résurgence de ce sens autrefois usité (voir note 18).

L'équivalent occasion semble pouvoir remplacer opportunité dans la majorité des contextes relevés ci-dessus. Parmi les acceptions relevées dans le corpus lexicographique, celles de "possibilité » et de « circonstance favorable ou qui vient à propos » recouvrent à la fois les sens français et les ES pour opportunité. Occasion s'utilise cependant dans d'autres contextes où il prend la valeur neutre de « circonstance», comme en (14):

(14) Le plaignant a notamment décrit avec un certain amusement des occasions où lui et l'accusée ont failli se faire prendre les culottes baissées par le mari de cette dernière, entre 2002 et 2004. (C-Jr 2012)

De plus, alors que occasion peut être accompagné de compléments nominaux ou adjectivaux « négatifs» (occasion de vengeance, mauvaise occasion, etc.), ce genre de complément n’a pas été relevé pour opportunité. Cette différence n'est cependant pas particulièrement marquée dans les corpus de productions textuelles. Une étude plus vaste serait nécessaire pour déterminer dans quelle mesure occasion est vraiment «équivalent » à opportunité compte tenu de la valeur de plus en plus positive que véhicule ce dernier.

\subsection{Supporter}

Au total, 1498 occurrences de supporter ont été analysées. Globalement, supporter est principalement utilisé dans des sens français tels « porter par en dessous », « subir avec patience » ou « tolérer », comme dans l'exemple (15):

19 Notons au passage que la fréquence relative (x/1 000000 mots) de opportunité dans le C-Jr passe de 10,5 en 1992 à 22,1 en 2012, ce qui fait que ce mot est proportionnellement deux fois plus présent dans les journaux en 2012 qu'en 1992. Beeching (2010) avance que l'augmentation de la fréquence d'un mot est généralement corrélée à un changement sémantique. 
(15) Le gouvernement Harper, qui ne supportait aucune contestation dans ce dossier, rétorquait avec agressivité aux questions de l'opposition [...]. (C-Jr 2012).

On note toutefois une différence de fréquence marquée entre les usages soignés du C-Jr et ceux, spontanés, du C-FB où les sens français ne comptent que pour $16,1 \%$ des occurrences (tableau 7 ).

ENCOURAGER, dont l'attestation lexicographique remonte à 1880, est le deuxième ES en fréquence dans le C-FB (37,5\%) et le plus usité dans le C-Jr. Au plan sémantique, cet $\mathrm{ES}$ présente un renversement axiologique complet par rapport aux sens français « tolérer » et « subir avec patience ». Les configurations lexicales dans lesquelles sont relevées ces acceptions ne présentent pourtant pas de différence (supporter + qqn) : l'interprétation positive de ENCOURAGER se déduit uniquement à partir du contexte d'utilisation. De plus, et bien que la description lexicographique de cet ES le situe principalement dans le domaine sportif, on le relève plus fréquemment dans des contextes sans lien avec le sport, comme en (16):

(16) J'espère qu'elle va vite se rétablir. On t'aime Dodo. Tout le Québec te supporte! (C-FB)

Lorsque le complément d'objet direct est une personne, la négation n'est presque jamais employée avec cet ES (trois occurrences seulement), sans doute parce que la construction ne pas supporter + qqn est ambiguë et porte à être interprétée avec le sens de " [ne pas] tolérer».

L'ES ADHÉRER est le sens le plus fréquent dans le C-FB avec 42,7 \% des occurrences, alors qu'il ne dépasse pas $10 \%$ dans le C-Jr. Il se caractérise par le fait que le soutien apporté est d'ordre moral ou idéologique : on supporte les actions d'une personne, ses idées ou ses prises de position politique, comme dans l'exemple (17):

(17) Le Syndicat des employées et employés de l'Université Laval (SEUL) supporte le recteur Brière et dénonce l'attitude de la FEUQ. (CJr 2012)

ADHÉRER apparaît très rarement (une seule occurrence) en construction négative, sans doute pour la même raison d'ambiguïté potentielle que pour ENCOURAGER.

L'emploi de l'ES POURVoIr est marginal : 0,9\% de l'ensemble des occurrences, et il est absent du C-Jr 2012. Cet ES partage avec le sens français « prendre en charge » une axiologie plutôt négative en lien avec la notion de fardeau, mais s'en distingue par le fait que son complément est un animé (enfant, parent) plutôt qu'un inanimé (dette, frais, charge, etc.), comme dans l'exemple (18) : 
Changements sémantiques sous l'influence de l'anglais...

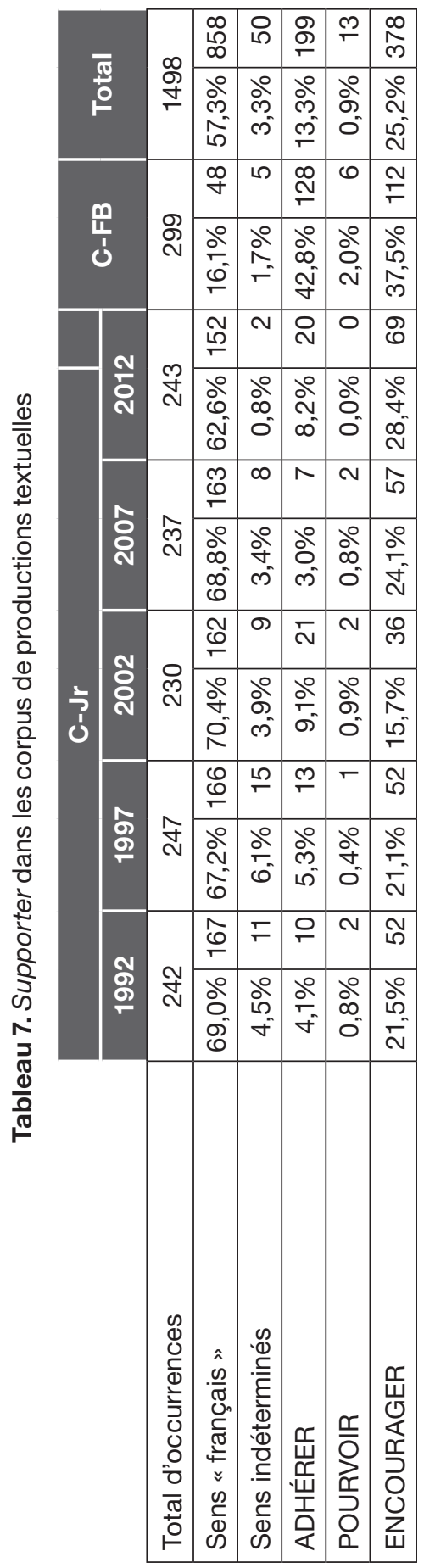


(18) [...] pis ca fait un sale boutte que je suis "contribuable" et que je supporte 3 générations avec une seule paye! alors....trouve autre chose a me dire que "va travailler". (C-FB)

Ainsi, des trois ES relevés pour supporter, seuls ENCOURAGER et ADHÉRER sont présents de manière significative dans les corpus $(25,2 \%$ et $13,3 \%$ respectivement). Ils s'utilisent dans les mêmes configurations lexicales que les sens en français « tolérer » et "subir avec patience ", alors même qu'ils sont de valeur opposée. Cette ambiguïté de sens est peut-être ressentie par les locuteurs, qui semblent éviter d'utiliser les deux ES dans des constructions en ne pas pouvant favoriser une interprétation axiologiquement négative. Il paraît à première vue difficile de voir la continuité sémantique entre les sens « français » et « anglais » de supporter compte tenu de cette opposition de valeur. Il faut, pour la saisir, tenir compte de changements de sens bien antérieurs à la période examinée dans l'étude, ce qui explique sans doute l'absence de véritables tendances diachroniques dans les corpus sondés ${ }^{20}$. Deux branches d'acceptions différentes, issues du latin sub- «sous » et portare « transporter", se rencontrent dans les sens français et anglais relevés : la première concerne les sens qui gravitent autour de la notion d'un mouvement de bas en haut (12 $2^{\mathrm{e}}$ siècle), tandis que la deuxième concerne les sens qui s'articulent autour de l'idée de subir quelque chose de pénible $\left(14^{\mathrm{e}} \text { siècle }\right)^{21}$. Les trois ES se rattachent à la première branche; il est d'ailleurs permis de mettre en doute l'influence de l'anglais comme facteur principal de la présence de ces acceptions en français au Québec. En effet, puisque les sens « appuyer qqn ", "prendre le parti de qqn » et « subvenir aux dépenses de qqn » sont tous attestés en France jusqu'au $18^{\mathrm{e}}$ siècle au moins et que les ES ADHÉRER et POURVOIR sont présents dans la lexicographie corrective québécoise dès la première moitié du $19^{\mathrm{e}}$ siècle ${ }^{22}$, on peut émettre l'hypothèse que ces sens ne sont jamais sortis des usages dans cette ancienne colonie. Dans tous les cas, l'ambiguïté sémantique que l’on observe aujourd'hui pour supporter est présente en français dès le $14^{\mathrm{e}}$ siècle.

20 Les fluctuations de fréquence pour supporter paraissent résulter davantage de la taille de l'échantillon analysé que de véritables changements sémantiques.

21 Dictionnaire historique de la langue française, sous supporter.

22 Ils sont mentionnés dans l'un des premiers ouvrages lexicographiques publiés au Québec: T. Maguire, Manuel des difficultés les plus communes de la langue française, adapté au jeune âge et suivi d'un Recueil de locutions vicieuses, Québec: L. Fréchette et Cie, 1841. Cet ouvrage ne faisait cependant pas partie du corpus lexicographique exploité. 
L'équivalent le plus fréquemment proposé pour éviter l'emploi de supporter dans un sens " anglais » est appuyer. De fait, sa description lexicographique comporte de nombreuses similitudes avec les sens «français» de supporter, et il peut remplacer tant ENCOURAGER que ADHÉRER. La principale différence semble résider dans la possibilité d'utiliser appuyer dans des constructions négatives comme en (19), là où supporter serait généralement évité afin de ne pas introduire d’ambiguïté sémantique :

(19) Pour une fois des gens se tiennent devant Charest sans rampé $<$ sic $>$ et se prosterner devant ce manipulateur pourris $\langle$ sic $>$. J'aurais honte de moi si je n’appuyais pas les étudiants (C-FB)

Globalement, les deux mots s'utilisent dans les mêmes contextes, comme dans les exemples (20) et (21) :

(20) Il n'est pas un centralisateur autoritaire, n'a jamais jeté l'anathème sur le nationalisme québécois, supportait l'accord du lac Meech et la loi 101. (C-Jr 2007)

(21) Il a témoigné au comité à titre personnel. Il appuyait le projet de loi. (C-Jr 2012)

Ainsi, les données des corpus de productions textuelles tendent à confirmer l'« équivalence » de appuyer et de supporter lorsque ce dernier est utilisé aux sens de ENCOURAGER et de ADHÉRER.

\subsection{Versatile}

Le mot versatile est très rare dans les productions textuelles malgré sa présence importante dans la lexicographie corrective ${ }^{23}$ : on n'en relève en effet que 179 occurrences sur les 421,5 millions de mots que comptent les deux corpus sondés (tableau 8). L’acception française « inconstant » ne représente que 12,8\% de ce total, avec toutefois un maximum de $22,7 \%$ dans le C-Jr 2002, dont l'exemple (22) est tiré :

(22) Il ne faut pas brusquer les humains. Ce sont des créatures versatiles, et pointilleuses. Ni les prendre par surprise ; leur esprit tourne comme le vent d'orage. [C-Jr 2002]

23 Versatile est décrit comme un emprunt sémantique dans 22 des 43 ouvrages québécois du corpus. 


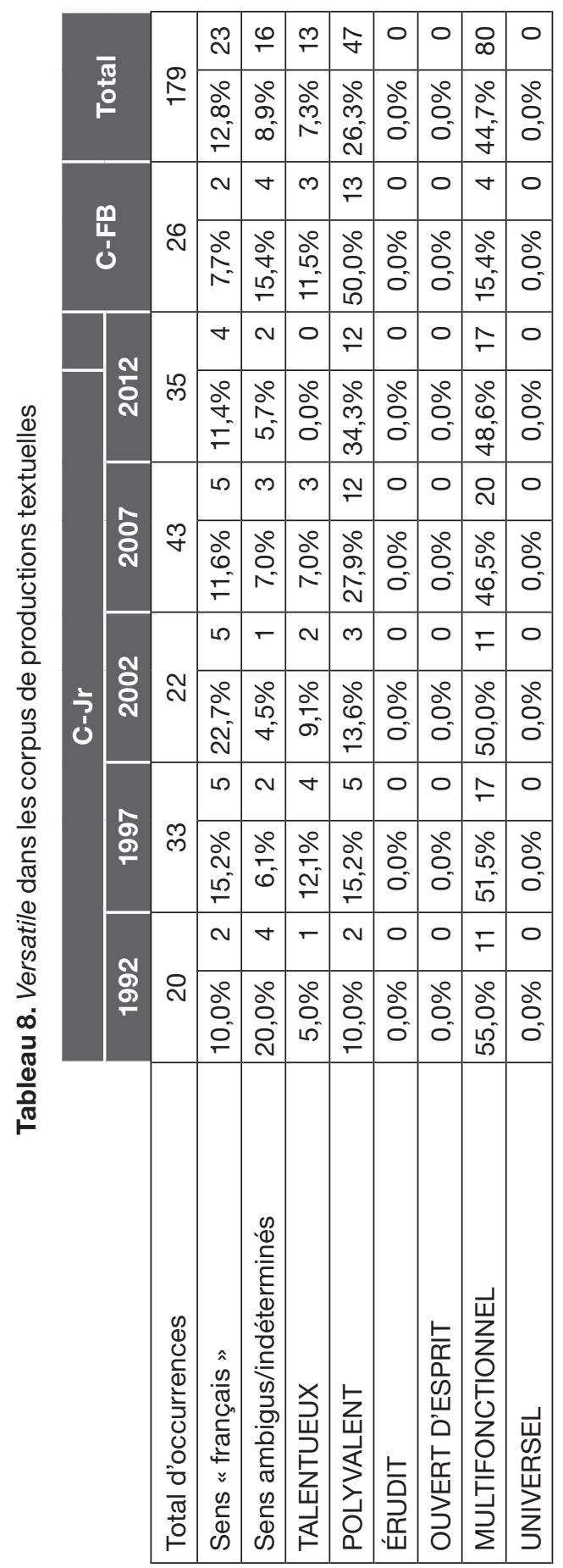


Des six ES relevés dans le corpus lexicographique, ÉRUDIT, OUVERT D'ESPRIT et UNIVERSEL ne sont pas attestés dans les productions textuelles. L'ES TALENTUEUX, qui se caractérise au plan sémantique par une axiologie fortement positive et qui sert à qualifier une personne qui démontre un talent marqué dans plusieurs domaines, ne compte que 13 occurrences (7,3\%), tel l'exemple (23) :

(23) De plus, l'image de Hello Kitty a toutes les qualités que l'on attend de la femme asiatique traditionnelle : pure, in nocente, cute, obéissante, tranquille, versatile, etc. (C-Jr 2002)

Le deuxième sens en termes de fréquence est polyvalent (26,3\%). Décrit pour la première fois en 2003 dans le corpus lexicographique, cet emploi est en croissance dans le C-Jr au cours de la période 1992-2012 et il représente la moitié des occurrences dans le discours spontané du C-FB. Cet ES à l'axiologie positive est utilisé pour qualifier une personne qui démontre de l'aptitude à accomplir des tâches de natures différentes, comme dans l'exemple (24):

(24) C'est un joueur versatile, capable de jouer autant sur la ligne défensive qu'à l'attaque. (C-Jr 2012)

L'ES le plus usité dans les productions textuelles est MULTIFONCTIONNEL, qui compte pour près de la moitié de l'ensemble des occurrences (44,7\%). Au plan sémantique, il se distingue à la fois du sens français et des autres ES relevés par le fait qu'il caractérise un objet, apprécié pour sa polyvalence, comme dans l'exemple (25):

(25) Afin de rationaliser les dépenses de cette nouvelle garde-robe, il faut - une fois de plus - miser sur des pièces versatiles pour ne pas dire caméléons. (C-Jr 1992)

Cet ES apparaît parfois dans des contextes où versatile est utilisé pour traduire et expliquer l'emploi du mot anglais versatile, notamment dans le C-Jr 1997, soit peu après la mise en marché de la nouvelle technologie DVD (Digital Versatile Disk). Le fait qu'il soit nécessaire d'expliciter le sens de ce mot montre à quel point il est alors peu répandu.

Une proportion non négligeable d'occurrences reste de sens «indéterminé » $(8,9 \%)$, tel l'exemple (26) :

(26) L'anglais est utiliser $<$ sic $>$ partout dans le monde, c'est une langue MONDIAL $<$ sic $>$, nettement plus facile à apprendre que le français, oui il est moins versatile que le français, mais les gens ont le choix de prendre et d'apprendre la langue qu'ils veulent. (C-FB) 
Le sens que donne l'auteur de ces lignes au mot versatile est pour le moins ambigu, puisque l'anglais y est décrit comme une «langue mondial» [sic] mais «moins versatile » que le français. Concrètement, compte tenu du fait que ces sens indéterminés représentent une proportion élevée des occurrences et que plusieurs sens « anglais » sont décrits dans le corpus lexicographique sans pour autant être relevés dans les productions textuelles ${ }^{24}$, le portrait d'ensemble obtenu suggère une signification très " élastique " pour versatile ${ }^{25}$. Sa très faible fréquence dans les usages tant soignés que spontanés en français au Québec fait en sorte que les locuteurs ont peu d'occasions de se familiariser avec la signification de ce $\operatorname{mot}^{26}$, qui reste donc sans doute assez vague pour une partie d'entre eux. Dans ces circonstances, l'influence des contextes où versatile sert à traduire l'anglais versatile peut être déterminante dans leur expérience lexicale.

Les trois ES attestés dans les productions textuelles, soit TALENTUEUX, POLYVALENT et MULTIFONCTIONNEL, partagent avec le sens français l'idée d'un changement. Mais alors que le changement qui touche à l'acception française se caractérise par l'instabilité (« inconstance »), celui qui touche les ES se caractérise par l'adaptabilité. Il s'agit d'une interprétation différente de la même notion qui mène à une opposition au plan axiologique, mais sans pour autant que l'ambiguité sémantique qui en résulte en soit nécessairement une pour les locuteurs, pour qui ces sens opposés ne cohabitent peut-être pas.

L'équivalent proposé pour remplacer versatile utilisé dans un ES est polyvalent. Au regard de la description lexicographique qui en est faite et des usages relevés dans les corpus, il apparaît que polyvalent s'emploie de manière semblable à versatile lorsque ce dernier est utilisé dans l'un des trois ES présentés ci-dessus, comme dans les exemples (27) et (28) :

(27) Le Twin Otter est reconnu pour être un appareil versatile qui peut opérer sous diverses conditions. (C-Jr 2012)

(28) [...] la XV Crosstrek se veut une voiture polyvalente élégante, capable d'affronter des chemins forestiers tout autant que le trafic de $17 \mathrm{~h}$ dans un centreville bondé. (C-Jr 2012)

Cet équivalent possède l'avantage d'être un mot très fréquent, dont la signification ne pose pas spécialement problème.

24 Pas moins de 37 équivalents différents sont proposés dans ce corpus.

25 Cette «souplesse» sémantique se traduit d'ailleurs par la présence importante de commentaires à connotation autonymique (cf. Julia 2001; Authier-Revuz 2003) dont l'objectif est de «fixer le sens » de versatile. Cet aspect ne peut être développé ici, faute d'espace.

$26 C f$. le concept d'historicité personnelle de Nyckees (2007). 


\section{Conclusion}

L'analyse comparative des corpus lexicographiques et de productions textuelles pour les mots alternative, opportunité, supporter et versatile a permis de montrer que, mis à part le cas de supporter, les différents sens « anglais » comptent pour la majorité des occurrences relevées, avec pour résultat que les sens «français » sont maintenant minoritaires dans les usages. Même si les ES sont globalement plus fréquents dans le discours spontané du C-FB, ils sont aussi d'usage généralisé dans le discours soigné du C-Jr. Il ressort également que certains ES décrits dans la lexicographie ne sont pas attestés dans les corpus sondés, tandis que d'autres sens, encore non décrits, sont de plus en plus utilisés.

Plutôt que de mettre en évidence un mécanisme unique d'« emprunt» de sens ponctuel et localisable permettant aux sens anglais de faire sens malgré les conflits sémantiques avec les acceptions françaises, les données du corpus montrent que plusieurs processus de diversification et d'évolution sémantique sont à l'œuvre. Les traces du renversement axiologique constaté dans l'usage actuel de alternative et de opportunité peuvent s'observer dans les changements sémantiques presque imperceptibles qui accompagnent des contextes d'utilisation de plus en plus positifs, en phase avec les valeurs de liberté et d'action individuelle de la société occidentale moderne. Lopposition apparente entre les acceptions «françaises » de supporter et certaines autres partageant des caractéristiques sémantiques avec le verbe de même étymon en anglais est quant à elle très ancienne ; les locuteurs semblent contourner l'ambiguïté sémantique provoquée par deux branches d'acceptions différentes en évitant généralement les constructions négatives (ne pas + supporter). Pour ce qui est de versatile, la rareté du mot fait en sorte que sa signification reste globalement floue pour la plupart des locuteurs, qui interprètent la notion de changement qu'elle comporte en fonction de leurs différentes expériences du lexique, où l'anglais (via l'apparition de nouvelles technologies) peut avoir joué un rôle non négligeable.

Les résultats de l'analyse montrent que dans le cas de alternative, l'équivalent solution ne permet pas de véhiculer l'axiologie très positive des sens influencés de l'anglais, un constat qui s'applique également mais dans une moindre mesure à occasion comme équivalent de opportunité. En ce qui concerne supporter et versatile, les équivalents appuyer et polyvalent semblent pouvoir s'utiliser dans le même genre de contextes sans différence sémantique significative.

Dans tous les cas, l'examen de l'utilisation des quatre mots permet de mettre en lumière le fait que les sens « anglais » ne sont pas complètement «étrangers » et qu'ils se rattachent tous à des notions présentes dans des acceptions fran- 
çaises. En ce sens, il nous apparaît nécessaire de repenser le concept d'« emprunt » sémantique, tant au regard de la diversification sémantique observée que de la difficulté, voire l'impossibilité, de localiser et de déterminer le moment de l'acte d'emprunt d'un sens. Le fait que dans le français utilisé au Québec existent, pour chacun des mots analysé ici, des mots de même famille ${ }^{27}$ utilisés également dans des sens partageant des caractéristiques sémantiques avec l'anglais semble être un argument supplémentaire en faveur de la nécessité d'une véritable théorisation de l'influence sémantique interlingue.

\section{Références bibliographiques}

Auroux Sylvain, 1994, La révolution technologique de la grammatisation, Liège, Mardaga Éditeur.

Authier-Revuz Jacqueline, 2003, « Le fait autonymique : langage, langue, discours. Quelques repères », in Parler des mots : le fait autonymique en discours, Jacqueline Authier-Revuz, Marianne Doury \& Sandrine Reboul-Touré (éds), Paris, Presses de la Sorbonne Nouvelle, p. 67-96.

Beeching Kate, 2010, "Semantic change : evidence from false friends ", Languages in contrast 10-2, p. 139-165.

Bouchard Chantal, 1998, La langue et le nombril. Histoire d'une obsession québécoise, Saint-Laurent, Fides.

Chamizo Dominguez Pedro, Nerlich Brigitte, 2002, «False friends : their origin and semantics in some selected languages », Journal of pragmatics 34 , p. $1833-1849$.

Colpron Gilles, 1970, « Divers déplacements de sens dans les anglicismes sémantiques ", Meta 15-1, p. 36-39.

Courbon Bruno, 2007, « Une réutilisation possible du concept d'usage en sémantique diachronique? ", in Le vocabulaire scientifique et technique en sciences du langage. Coldoc 2007, Julie Glikman, Leda Mansour \& Stéphanie Weiser (éds.), p. 102-128, 2012, «Intégration syntagmatique du sens lexical et établissement de rapports synonymiques ", in La synonymie, Françoise Berlan \& Gérard Berthomieu (éds), Paris, Presses de l'Université Paris-Sorbonne, p. 329-341.

Courbon Bruno, Paquet-Gauthier Myriam, 2014, « Faux amis / vrais ennemis : réutilisations de la notion d'anglicisme dans le discours métalinguistique au Québec ", Le discours et la langue 6-1, 'Métalangage et expression du sentiment linguistique «» profane », Michelle Lecolle (coord), p. 143-173.

27 Dont alternatif, opportuniste, support et versatilité, pour ne citer que ceux-là. 
Darbelnet Jean, 1986, « Réflexions sur la typologie de l'emprunt linguistique et des situations bilingues ", Multilingual Journal of Cross-Cultural and Interlanguage Communication 5-4, p. 199-204.

De Villers Marie-Éva, 2005, Le vif désir de durer, Montréal, Québec Amérique.

Dictionnaire de l'Académie française, $1^{\mathrm{e}}$ éd. (1694), $4^{\mathrm{e}}$ éd. (1762), $5^{\mathrm{e}}$ éd. (1798), $6^{e}$ éd. (1835), $8^{e}$ éd. (1932-1935) et $9^{e}$ éd. (1992-), numérisées et mises en ligne par l'ARTFL \& le CNRTL, http://www.cnrtl.fr/dictionnaires/anciens/.

Dictionnaire historique de la langue française, 2012, Alain Rey (éd.), Dictionnaires le Robert, Paris.

Dion-Girardeau Samuel, 2013, «Les pronoms personnels en français québécois : utilisation d'un corpus écrit non standard », présentation faite aux XXVII ${ }^{e s}$ Journées de linguistique (Université Laval, 13-15 mars 2015).

Escayola Ines, 2000, Étude quantitative et qualitative de 45 emprunts de sens dans sept types de discours québécois, Mémoire de maîtrise, Université de Sherbrooke (Canada).

Humbley John, 1974, «Vers une typologie de l'emprunt linguistique », Cahiers de lexicologie 25-2, p. 46-70.

Julia Catherine, 2001, Fixer le sens? La sémantique spontanée des gloses de spécification du sens, Paris, Presses de la Sorbonne Nouvelle.

Klein Jean-René, Lienart Nathalie, Ostyn Stéphane, 1997, « L’anglicisme et la presse : Enquête et analyse à travers quatre quotidiens français et belges ", Revue de Linguistique Romane 61-243-244, p. 337-360.

Lahire Bernard, 2004, La culture des individus : dissonances culturelles et distinction de soi, Paris, Éditions La Découverte.

Lamontagne Linda, 1996, La conception de l'anglicisme dans les sources métalinguistiques québécoises de 1800 à 1930, Québec, CIRAL.

Loubier Christiane, 2011, De l'usage de l'emprunt linguistique, Montréal, Office québécois de la langue française.

Mareschal Geneviève, 1994, «Étude typologique et comparative de l'anglicisation et des anglicismes dans quatre aires de la francophonie », in Actes du colloque sur les anglicismes et leur traitement lexicographique, Maurice Pergnier (éd.), Québec, Office de la langue française, p. 25-37.

Martel Claude, Cajolet-Laganière Hélène, Langlois Marie-France, 2001, « Les textes journalistiques québécois sont-ils "envahis" par les emprunts critiqués à l'anglais? ", Terminogramme 97-98, p. 47-71.

Merriam-Webster Online Dictionary, Encyclopaedia Britannica Company. En ligne, édition mise à jour en continu : http://www.merriam-webster.com/. 
Multidictionnaire de la langue française, 2009, Marie-Éva de Villers, Montréal, Québec Amérique.

Nouveau Petit Robert de la langue française, 2009, Alain Rey (éd.), Paris, Dictionnaires Le Robert.

Nyckees Vincent, 2006, «Rien n'est sans raison : les bases d'une théorie continuiste de l'évolution sémantique », in Aspects diachroniques du vocabulaire, François Gaudin \& Danielle Candel (éds.), Mont-Saint-Aignan, Presses Universitaires de Rouen et du Havre, p. 15-88, 2007, « La cognition humaine saisie par le langage : de la sémantique cognitive au médiationnisme», Corela HS-6.

Oxford English Dictionary Online, Oxford University Press. En ligne, édition mise à jour en continu: www.oed.com.

Paquet-Gauthier Myriam, 2015, Sens influencés de l'anglais en français au Québec : utilisation, perception et intégration, Mémoire de maîtrise, Université Laval.

Poirier Claude, 1994, «L'anglicisme dans les dictionnaires de langue québécois : le point de vue de l'équipe du Trésor de la langue française au Québec", in Actes $d u$ colloque sur les anglicismes et leur traitement lexicographique, Montréal, Office de la langue française, p. 223-246.

Sablayrolles Jean-François, 2016, «Emprunts et influences d'autres langues », in Emprunts néologiques et équivalents autochtones en français, en polonais et en tchèque, Zuzana Hildenbrand, Alicja Kacprzak et Jean-François Sablayrolles (éds.), Limoges, Lambert-Lucas, p. 23-35.

Trésor de la langue française informatisé, Bernard Quemada \& Paul Imbs (éds.), édition numérisée et mise en ligne par le CNRTL, http://atilf.atilf.fr/dendien/ scripts/tlfiv4/showps.exe? $\mathrm{p}=$ combi.htm;java=no.

Valdman Albert, Auger Julie, Piston-Hatlen Deborah, 2005, «Introduction », in Le français en Amérique du Nord. État présent, Albert Valdman, Julie Auger \& Deborah Piston-Hatlen (éds.), Sainte-Foy, Les Presses de l'Université Laval, p. 1-35.

Walker James, 2013, Les attitudes envers les anglicismes. Une étude sociolinguistique des emprunts dans différentes communautés francophones, Lille, Atelier national de reproduction des thèses. 